\title{
Passive propulsion in turbulent flows
}

\author{
J. Yang, M. Davoodianidalik, H. Xia, H. Punzmann, M. Shats, and N. Francois* \\ Research School of Physics and Engineering, Australian National University, Canberra, \\ Australian Capital Territory 2601, Australia
}

(Received 20 December 2018; published 25 October 2019)

\begin{abstract}
The ability of a device to exploit the energy of a flow to generate thrust is the main feature of passive propulsion. In a turbulent flow, such energy conversion is challenging due to the unpredictable and disordered fluid motion. In wave-driven turbulence, it has recently been demonstrated that asymmetric floating rotors can tap the energy of ambient fluctuations to fuel directed rotation. Here we report on the dynamics of asymmetric floating vehicles capable of passively propelling themselves in two-dimensional turbulence. We show experimentally how the shape of a floater and its rotational dynamics conspire to allow harvesting energy of the turbulent fluid motion. The translation and rotation of the floater are shown to be strongly coupled. The propulsion velocity and the rotational diffusion timescale depend on the relative size of the floating vehicle with respect to the turbulence forcing scale. The geometry of the floater is investigated in the range of circular-sector-shaped objects and a shape optimizing its propulsion is identified. At times larger than the rotational diffusion timescale, our results shed light on a substantial increase of the turbulent diffusion coefficient of anisotropic objects due to the coupling between propulsion and rotational diffusion.
\end{abstract}

DOI: 10.1103/PhysRevFluids.4.104608

\section{INTRODUCTION}

The generation of thrust in a fluid flow is an important problem in fluid mechanics with many applications in aerodynamics, wind and wave energy, as well as biology [1-7]. Propulsion in a flow can be achieved via various mechanisms which are broadly classified as active or passive to distinguish whether or not it involves the use of the internal energy of a device (by heat engines, electric motors, etc.). Passive mechanisms are usually hard to characterize because they rely on a complex fluid-structure coupling [3,8-12]. There has been research into the passive generation of thrust by a foil in various flows [13,14]. This topic also resonates with research carried out on the hydrodynamics of aquatic creatures where passively generated thrust (with no muscular activity) is referred to as passive swimming $[8,14]$. The advantage of passive over active propulsion mechanisms is that no energy needs to be expended by the swimmer to generate locomotion. An interesting aspect of passive locomotion concerns the ability of an object to efficiently propel itself in the presence of unsteady flows, in particular turbulent flows $[14,15]$.

The description of passive propulsion in turbulence remains an open question which requires insights from experimental studies. A better understanding of the phenomenon relies on the detailed knowledge of the coupling of passive objects with turbulent flows. The dynamics of passive objects advected in any flow depends on the object density, its size, and its shape. The description of this dynamics is particularly challenging in turbulent flows. Recent experiments clarified some important features of the turbulent transport of inertial and/or anisotropic particles [16,17]. In wave-driven

*nicolas.francois@anu.edu.au 
two-dimensional turbulence, a floating object can exploit the fluid erratic motion to fuel either directional propulsion or rotation [18]. It was shown that wave-driven turbulence possesses an underlying fabric which consists of riverlike structures [18-20]. The motion of a floating object can be strongly coupled with this flow fabric, if its shape is asymmetric. It was demonstrated that the kinetic energy contained in the turbulent eddies can be rectified to power either a chiral rotor or a self-propelled asymmetric vehicle. The case of a turbulence-driven rotor has been studied in detail in Ref. [18], while the case of self-propelled objects remains unexplored.

Here we report on asymmetric objects capable of passively propelling themselves at the airwater interface by tapping into the energy of wave-driven turbulent flows. It is shown that the key mechanism behind the turbulence-driven propulsion is the coupling between the translation and rotation of the object. More precisely, we demonstrate experimentally how the asymmetric shape of an object and its slow rotational dynamics conspire to allow harvesting the energy of turbulence. Both the propulsion velocity $V_{p}$ and the characteristic time $T_{\theta}$ of rotational diffusion depend on the relative size of the object with respect to the turbulence forcing scale $L_{f}$. This size dependence highlights the role played by the underlying fabric of the wave-driven turbulence in the rectification phenomenon. The geometry of the floater is also investigated and a shape optimizing its propulsion is identified. Our results suggest a different mechanism of propulsion at the air-water interface [21] and reveal a regime of enhanced turbulent transport for anisotropic objects due to the coupling between propulsion and rotational diffusion.

\section{EXPERIMENTAL RESULTS}

In these experiments, turbulent flows are produced on a liquid surface perturbed by parametrically excited waves, also known as Faraday waves [22]. This is a convenient experimental system to produce erratic surface flows that in many respects resemble two-dimensional turbulence [23-28]. The waves are generated in a circular 290 -mm-diam container shaken vertically at a frequency set in the range of $f_{s}=30-120 \mathrm{~Hz}$ (see more details in the Supplemental Material [29]). Although energy is injected into the vertical oscillations of Faraday waves, part of this energy is converted into chaotic horizontal fluid motion via the generation and interaction of horizontal vortices [27,30,31]. Those vortices have a characteristic size of $L_{f}$, equal to half the Faraday wavelength $\lambda$. An important feature of wave-driven turbulent flows is that the forcing scale $L_{f}=\lambda / 2$ is a function of the forcing frequency $f_{s}$. In the experiments, $L_{f}$ is tuned within the range $3-9 \mathrm{~mm}$. The wave-driven flows are random with no mean component. Transport properties of these flows have been extensively studied [25,28,32-35]. The timescale $T_{L}$ of the wave-driven flows (deduced from the Lagrangian velocity autocorrelation function) is typically of the order of $0.2 \mathrm{~s}$. The rms velocity $U$ of the turbulent flow depends on the kinetic energy accumulated over a broad range of scales. This range of wave numbers is illustrated in the kinetic energy spectrum in Fig. 1(a), where $k / k_{f}=1$ corresponds to the energy injection scale $L_{f}$. Such spectra are formed in the process referred to as the inverse energy cascade in two-dimensional (2D) turbulence [23]. They emerge due to a transfer of energy from the injection wave number $k_{f}$ towards smaller $k$ (larger scales). In Fig. 1(a), this energy transfer is identified by a spectrum scaling of $E_{k} \propto k^{-5 / 3}$, which is consistent with Kraichnan's theory of 2D turbulence [23]. Figure 1(b) illustrates the chaotic motion of tracer particles (50 $\mu \mathrm{m}$ diameter) at the surface perturbed by Faraday waves. Recent work uncovered that wave-driven turbulence possesses an underlying fabric which consists of riverlike structures [18]. When observed from the Lagrangian point of view, these structures are seen as coherent bundles of fluid particle trajectories. Figure 1(c) shows a coherent bundle measured using a combination of particle tracking velocimetry and a topological tool based on the braid theory $[18,34,36]$. The typical width of these riverlike streaks, or bundles, is comparable to $L_{f}$.

In this study, we are interested in the behavior of large floating objects placed in such wave-driven turbulent flows [see Fig. 1(b)]. The floating objects are printed on a high-resolution 3D printer and are made of a thermoplastic polymer (see more details in the Supplemental Material [29]). The floaters have various shapes with a "characteristic" radius $r_{s}$ in the range of $r_{s}=2.5-40 \mathrm{~mm}$. 

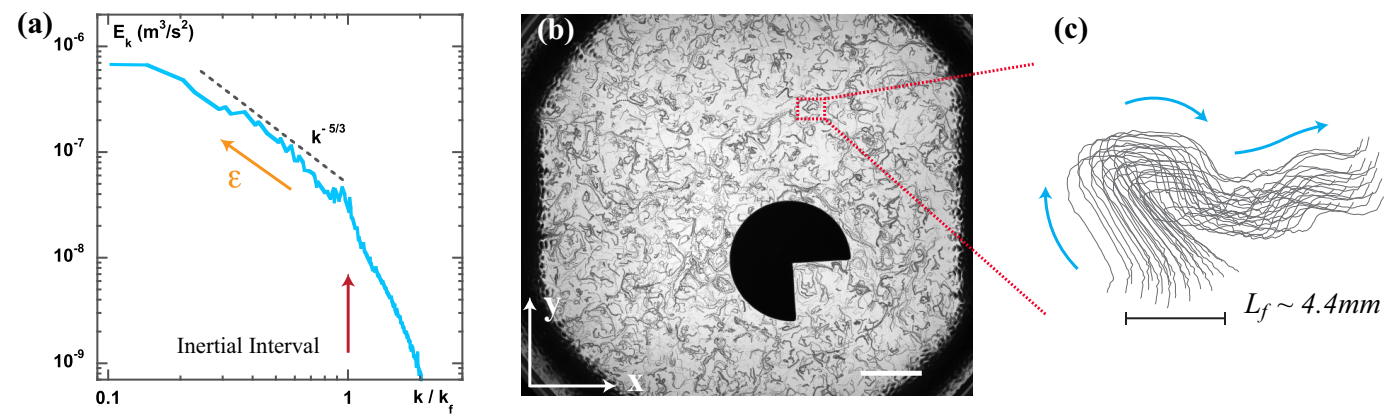

FIG. 1. Statistics and visualization of wave-driven turbulent flows. (a) Wave number spectrum $E_{k}(k)$ of the horizontal kinetic energy of the wave-driven flow. The red arrow indicates the forcing wave number $k_{f}$ at which energy is injected into the flow. The orange arrow indicates the direction of the energy flux $\epsilon$ towards small wave numbers (i.e., large scales). The $k^{-5 / 3}$ scaling signals the presence of the inverse energy cascade, a mechanism consistent with the Kraichnan theoretical prediction of 2D turbulence. (b) Fluid particle streaks around a 40-mm-radius anisotropic object. The dynamics of the floating object is much slower than that of the flow. Fluid particle streaks are tracked for $1 \mathrm{~s}$. The characteristics of the turbulent flow are $U=3 \times 10^{-2} \mathrm{~ms}^{-1}$, $L_{f}=4.4 \mathrm{~mm}$, and $T_{L}=0.15 \mathrm{~s}$. The scale bar is equal to $40 \mathrm{~mm}$. (c) From the Lagrangian viewpoint, wavedriven turbulence is composed of numerous interacting riverlike structures whose dynamics is studied using particle tracking. This figure shows such an elongated structure, or a bundle of trajectories, with a characteristic width determined by the forcing scale $L_{f}$. The bundle is tracked for a time interval of $8 T_{L}$; the blue arrows indicate the direction of the bundle motion.

\section{A. Passive swimming powered by turbulence}

Figures 2(a) and 2(b) show trajectories of floating objects of different shapes in wave-driven turbulent flows. These objects are larger than the characteristic length scale of the flow $L_{f}$. The motion of a floating circular disk is erratic, as can be seen in Fig. 2(a) (see the video in the Supplemental Material [29]). The main focus of this study is the behavior of noncircular floating objects, in particular disks with a cut-out $90^{\circ}$ sector. The motion of such floater in a turbulent flow is very different from that of a circular disk: It travels along an almost straight trajectory [Fig. 2(b)] (see video in Supplemental Material [29]). The direction of motion is always oriented along the bisector of the missing sector. Due to its ability to propel itself on a wavy fluid surface, we call this object a surfer. We study the behavior of surfers for different scale ratios $r_{s} / L_{f}$ at a fixed kinetic energy of turbulence $U^{2}$.

A typical experimental run produces 1000 independent trajectories for any given value of the ratio $r_{s} / L_{f}$. An important statistical characteristic of these trajectories is the mean-square displacement (MSD) computed as $\left\langle\delta r^{2}\right\rangle=\left\langle|\overrightarrow{\mathbf{r}}(t)-\overrightarrow{\mathbf{r}}(0)|^{2}\right\rangle$, where $\overrightarrow{\mathbf{r}}(t)$ is the position of the floating object in the horizontal plane at time $t$ and \langle\rangle denotes the statistical average. In the case of a floating disk [Fig. 2(c)], the MSD shows a transition between a ballistic regime at short times $\left(\left\langle\delta r^{2}\right\rangle \sim t^{2}\right.$ at $t \ll$ $\left.T_{\text {disc }}\right)$ to a diffusive behavior at longer times $\left(\left\langle\delta r^{2}\right\rangle=2 D t\right.$ at $\left.t \gg T_{\text {disc }}\right)$. Here $T_{\text {disc }}$ is the characteristic time obtained by integrating the Lagrangian velocity autocorrelation function $\rho_{u}(\Delta t)=\left\langle\mathbf{u}_{\mathbf{0}}\left(t_{0}+\right.\right.$ $\left.\Delta t) \mathbf{u}_{\mathbf{o}}\left(t_{0}\right)\right\rangle / u_{o}^{2}$, where $\mathbf{u}_{\mathbf{o}}$ is the velocity vector of the floating object and $u_{o}$ is its rms value; $\rho_{u}(\Delta t)$ characterizes the process of memory loss during the object's motion. In the case of a disk [Fig. 2(d)], $\rho_{u}(\Delta t)$ is a rapidly decaying function and $T_{\text {disc }}$ is typically of the order of a second. This behavior for the MSD and $\rho_{u}(\Delta t)$ is observed independently of the disk radius.

At large values $r_{s} / L_{f}>6$, the MSD of a surfer shows only a ballistic behavior, which is statistical evidence of the strong propulsive effect [see Fig. 2(c)]. The motion of the surfer is not purely deterministic but presents a certain level of randomness due to fluctuations in both its velocity magnitude and direction. This is illustrated in Fig. 2(b): The motion of a surfer is strongly biased towards one direction, but it also shows small erratic agitation under the action of the surrounding 

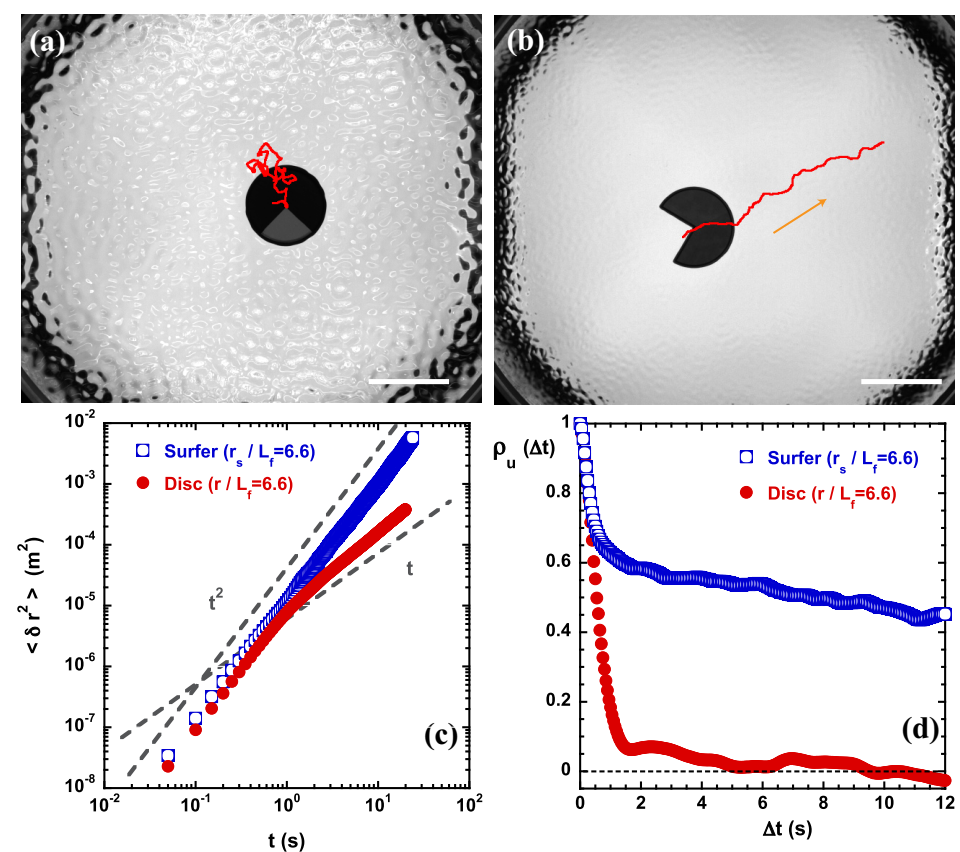

FIG. 2. Passive swimming powered by turbulence. (a) Trajectory (red line) of a floating disk (with a $90^{\circ}$ sector in gray) in wave-driven turbulence; the motion of the disk is tracked for $50 \mathrm{~s}$. (b) Trajectory (red line) of an anisotropic object, a surfer, in a wave-driven flow with the same turbulence intensity as in (a); the motion of the object is tracked for $25 \mathrm{~s}$. While the motion of a disk is erratic, the motion of the surfer is highly directional (the direction is indicated by the orange arrow). The scale bars are $40 \mathrm{~mm}$. (c) Mean-square displacement $\left\langle\delta r^{2}\right\rangle$ of a disk and of a surfer versus time. The gray dotted lines indicate the scalings expected for the ballistic and diffusive regimes. (d) Autocorrelation functions $\rho_{u}(\Delta t)$ of the velocity of a disk and that of a surfer. The data in (c) and (d) are averaged over 1000 independent trajectories of a disk and a surfer which have the same radius of $20 \mathrm{~mm}$. The parameters of the turbulent flow are $U=2.3 \times 10^{-2} \mathrm{~ms}^{-1}, L_{f}=3 \mathrm{~mm}$, and $T_{L}=0.15 \mathrm{~s}$.

turbulence. This dynamics is clearly reflected in the autocorrelation function $\rho_{u}(\Delta t)$, which shows two regimes [see Fig. 2(d)]: (i) a fast decrease of $\rho_{u}(\Delta t)$ at short timescales $\left(\Delta t<T_{\text {disc }}\right)$ similar to the one observed for a disk and linked to the turbulent fluctuations, followed by (ii) a much slower decrease related to the propulsion phenomenon.

The shape of $\rho_{u}(\Delta t)$ allows us to identify two components in the surfer's motion: the propulsive one and the turbulent one. There is in fact a competition between these two components: The magnitude of one compared to the other can be tuned by varying the ratio $r_{s} / L_{f}$. This results in clear changes in the behavior of the surfer [Figs. 3(a) and 3(b)]. Figures 3(c) and 3(d) show the MSD of a surfer with radius $r_{s}=20 \mathrm{~mm}$ for two different values of the flow characteristic scale $L_{f}$. It can be seen that the long-time behavior ( $t>T_{\text {disc }}$ ) of the MSD can be switched from a propulsion-dominated regime to a purely diffusive motion by decreasing the size ratio from $r_{s} / L_{f}=6.6$ to $r_{s} / L_{f}=2.5$.

\section{B. Orientational dynamics}

The propulsion effect is obviously connected to the surfer asymmetry and therefore to its orientation. To understand the origin of the slow dynamics detected in the velocity autocorrelation function [Fig. 2(d)], we now investigate the rotational dynamics of the surfers.

The surfer's orientation is characterized by the angle $\theta_{s}$ and the associated vector $\mathbf{n}_{\mathbf{s}}$ as shown in Fig. 4(a). We then measure the surfer's angular velocity $\omega(t)=\partial \theta_{s} / \partial t$ and compute the 

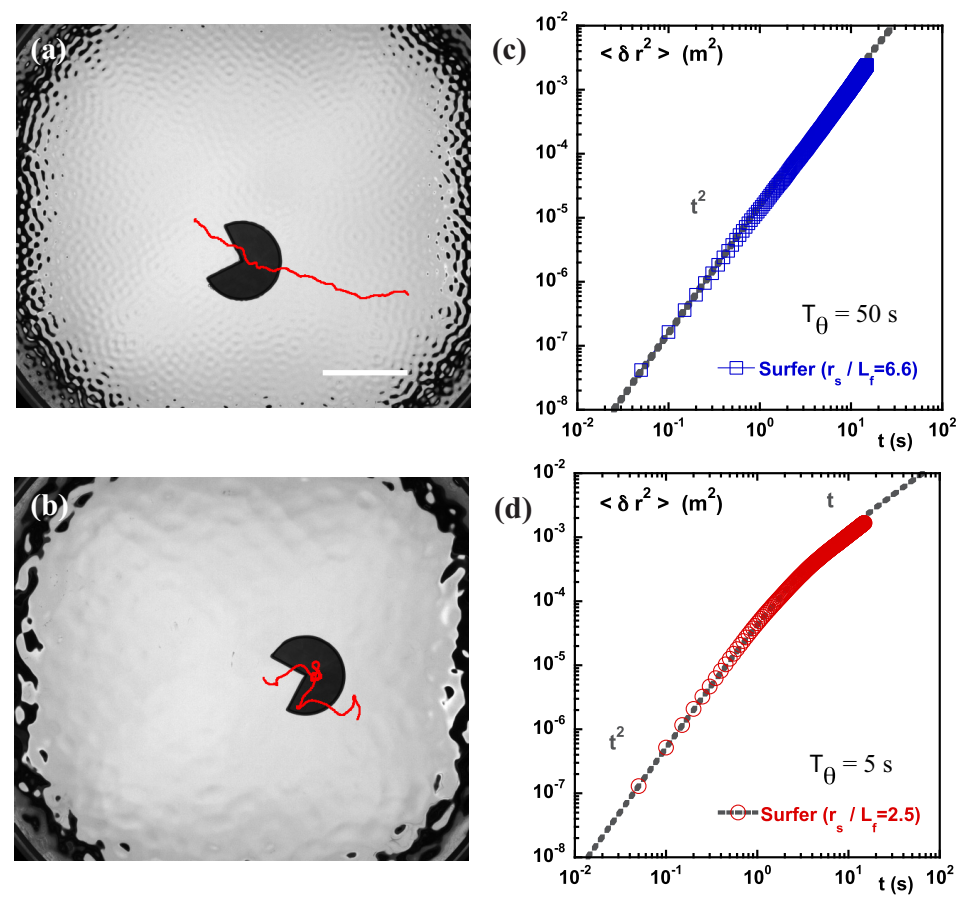

FIG. 3. (a) and (b) Trajectories (red lines) of the same surfer (radius $r_{s}=20 \mathrm{~mm}$ ) in two different turbulent flows having identical kinetic energy but different forcing scales $L_{f}$. In (a) the ratio $r_{s} / L_{f}=6.6$, while in (b) $r_{s} / L_{f}=2.5$; the surfer motion is tracked for $25 \mathrm{~s}$. (c) and (d) Mean-square displacement of the surfer versus time for the two different size ratios shown in (a) and (b). The data are averaged over 1000 independent trajectories of the surfer and gray dotted lines are fits based on Eq. (1) of Sec. II D. The turbulent flow parameters are $U=2.3 \times 10^{-2} \mathrm{~ms}^{-1}$ and (a) $L_{f}=3 \mathrm{~mm}$ and (b) $L_{f}=7.7 \mathrm{~mm}$.
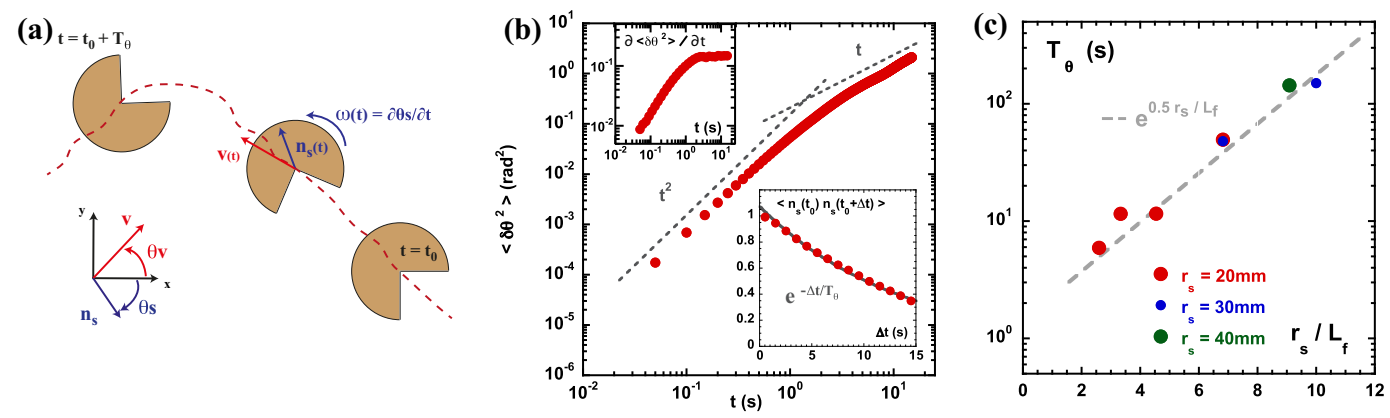

FIG. 4. Orientational dynamics of the surfer. (a) Angular coordinate system $\left(\theta_{s}, \theta_{v}\right)$ of a surfer; $\theta_{s}$ characterizes the surfer's orientation and $\theta_{v}$ characterizes the direction of the surfer's velocity. The unit orientation vector $\mathbf{n}_{\mathbf{s}}$ (associated with the angle $\theta_{s}$ ) is also defined. (b) Mean-square angular displacement (MSAD) $\left\langle\delta \theta^{2}\right\rangle(t)$ for the value of the ratio $r_{s} / L_{f}=3.3$. The top left inset shows the derivative of the MSAD with respect to time. The bottom right inset shows the autocorrelation function of the orientation vector $\mathbf{n}_{\mathbf{s}}$ of surfers for the same value $r_{s} / L_{f}$. The gray lines indicate an exponential fitting function $e^{-\Delta t / T_{\theta}}$. (c) Characteristic time $T_{\theta}$ of the rotational diffusion versus the ratio $r_{s} / L_{f}$. Here $T_{\theta}$ characterizes the randomization of the surfer's orientation. Experimental data can be fitted by $T_{\theta}\left(r_{s} / L_{f}\right) \sim\left(e^{0.5 r_{s} / L_{f}}\right)$. The experimental parameters are $r_{s}=20,30$, and $40 \mathrm{~mm}, U=2.3 \times 10^{-2} \mathrm{~ms}^{-1}$, and $T_{L} \approx 0.2 \mathrm{~s}$. 
mean-squared angular displacement $\left\langle\delta \theta^{2}\right\rangle=\left\langle\left[\sum \omega(t) \delta t\right]^{2}\right\rangle$. The latter presents a gradual transition from a ballistic $\left(\left\langle\delta \theta^{2}\right\rangle \propto t^{2}\right)$ to a diffusive regime $\left(\left\langle\delta \theta^{2}\right\rangle \propto t\right)$, which is identified in Fig. 4(b) (main panel and top inset). The diffusive rotation is characterized by a coefficient $D_{\theta}$ such that $\left\langle\delta \theta^{2}\right\rangle=2 D_{\theta} t$.

Under the action of turbulence, the surfer undergoes rotational diffusion with a characteristic timescale $T_{\theta}=1 / D_{\theta}$. Here $T_{\theta}$ characterizes the randomization of the surfer's orientation, i.e., the statistically averaged time it takes for the initial orientation $\theta_{s}\left(t_{0}\right)$ to be randomized; $T_{\theta}$ can also be directly estimated from the autocorrelation function of the orientation vector $\mathbf{n}_{\mathbf{s}}$ [see bottom right inset of Fig. 4(b)]. The timescale $T_{\theta}$ is strongly dependent on the ratio $r_{s} / L_{f}$. Figure 4(c) shows that $T_{\theta}$ increases exponentially with the increase in the ratio $r_{s} / L_{f}$ such that $T_{\theta} \approx e^{\gamma r_{s} / L_{f}}$. In these experiments, $T_{\theta}$ can be varied over almost two orders of magnitude. The exponential rate of growth $\gamma$ is of order unity and points to $L_{f}$ as the characteristic length scale that governs the rotational dynamics.

\section{Translation-rotation coupling}

In this section, we investigate the characteristics of the coupling between the rotation and translation of a surfer. First we study the correlations between the surfer's orientation $\theta_{s}$ and the direction of its velocity vector defined by the angle $\theta_{v}$ [see Fig. 4(a)]. Along a surfer's trajectory, the angle difference $\delta \theta_{s v}=\theta_{s}-\theta_{v}$ is a random variable with a clear bias due to the surfer asymmetric shape. This statistical bias is clearly seen in the probability density function (PDF) of the angle difference $\delta \theta_{s v}$, as shown in Fig. 5(a). In the case of a disk this PDF is flat, illustrating the absence of correlations between the velocity vector direction and an arbitrary direction marked on the disk. In contrast, the PDF becomes peaked around $\delta \theta_{s v}=0^{\circ}$ for a surfer and the effect is stronger as the ratio $r_{s} / L_{f}$ becomes larger. Given the shape of the PDF, we define three orientation domains colored in red, green, and blue, respectively, and labeled as domains $\Delta_{1}, \Delta_{2}$, and $\Delta_{3}$ in Fig. 5(b). The domain $\Delta_{1}$ corresponds to the propulsive events for which $\left\|\delta \theta_{s v}\right\| \in\left[0,+45^{\circ}\right], \Delta_{2}$ is the turbulent region for which $\left\|\delta \theta_{s v}\right\| \in\left[45^{\circ}, 135^{\circ}\right]$, and $\Delta_{3}$ is the anticorrelated domain for which $\left\|\delta \theta_{s v}\right\| \in\left[135^{\circ}, 180^{\circ}\right]$.

The magnitude of the surfer velocity modulus $\left|V_{s}\right|$ depends on the angle difference $\left\|\delta \theta_{s v}\right\|$. This effect is studied by computing the average $V_{s i}$ of the speed $\left|V_{s}\right|\left(\delta \theta_{s v}\right)$ over each orientational domain $\Delta_{i}$, i.e., $V_{s i}=\left\langle\left|V_{s}\right|\right\rangle_{\Delta_{i}}$. Figure 5(c) shows the average value $\left\langle\left|V_{s}\right|\right\rangle_{\Delta_{i}}$ normalized by the average speed of a disk as a function of the domain $\Delta_{i}$. The speed of a surfer becomes a function of its orientation for values of $r_{s} / L_{f}>3$ (while the conditional averaging of the disk speed shows no dependence on its orientation). As the ratio $r_{s} / L_{f}$ increases, the speed $\left\langle\left|V_{s}\right|\right\rangle_{\Delta_{1}}$ becomes larger than the speed measured for a disk of the same size as the surfer. Moreover, the speed component $\left\langle\left|V_{s}\right|\right\rangle_{\Delta_{1}}$ is larger than $\left\langle\left|V_{s}\right|\right\rangle_{\Delta_{2}}$ and $\left\langle\left|V_{s}\right|\right\rangle_{\Delta_{3}}$. We also note the nonmonotonic behavior of the ratio $\left\langle\left|V_{s}\right|\right\rangle_{\Delta_{3}} /\left\langle\left|V_{\text {disc }}\right|\right\rangle$ with respect to $r_{s} / L_{f}$. The previous results confirm that $r_{s} / L_{f}$ is a key parameter for the emergence of the directional motion.

The mean-square velocity $\left\langle V_{s}^{2}\right\rangle$ of a surfer is a decreasing function of the ratio $r_{s} / L_{f}$. This is observed in the whole range of size ratio $r_{s} / L_{f}$ studied, as shown in Fig. 5(d). The mean-square velocity of the larger surfer $\left(r_{s} / L_{f}=10\right)$ is almost two orders of magnitude smaller than that of a small surfer $\left(r_{s} / L_{f}<1\right)$. The kinetic energy of the surfer is directly measured as $E_{s}=M_{s}\left\langle V_{s}^{2}\right\rangle$, where $M_{s}$ is the mass of the surfer. Figure 5(e) reveals that the energy $E_{s}$ does not depend on the parameter $r_{s} / L_{f}$ when $r_{s} / L_{f}>1$. It turns out that large surfers, i.e., $r_{s} / L f>1$, extract the same amount of energy from the turbulent flow. When compared with the data shown in Figs. 5(a)-5(c), these results reveal that the main ingredient for strong propulsion is the partition of the surfer's kinetic energy $E_{s}$ between the different components $V_{s i}$ of its velocity.

To gain further insights into this phenomenon, we define the speed $V_{p}=p_{1} V_{s 1}-p_{3} V_{s 3}$, where $p_{i}$ is the probability of $\left\|\delta \theta_{s v}\right\| \in \Delta_{i}$. In the following, we will refer to $V_{p}$ as the propulsion speed. This allows us to compute a propulsion parameter $\beta$ defined as $\beta=V_{p} / p_{2} V_{s 2}$ which characterizes the partition of energy between the propulsive and turbulent components of the surfer's velocity. By construction, $\beta=0$ for a disk. The dependence of $\beta$ on the parameter $r_{s} / L_{f}$ is shown in Fig. 5(f). 
(a)

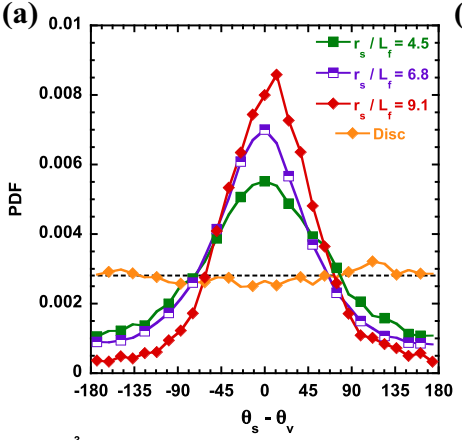

(d)

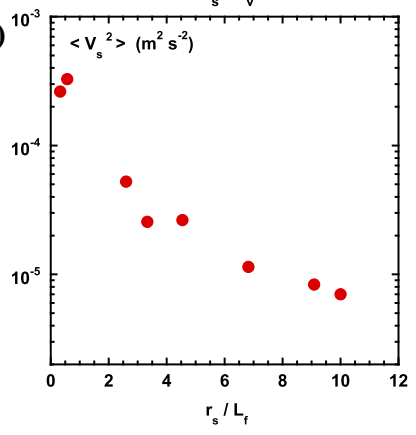

(b)
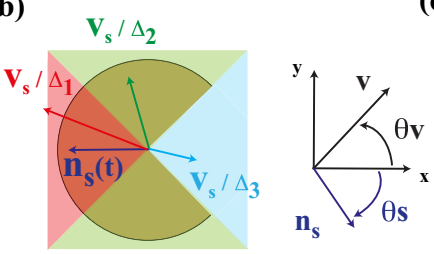

$\Delta_{1},\|\theta s-\theta v\| \in\left[0^{\circ}, 45^{\circ}\right]$

$\Delta_{2},\|\theta \mathrm{s}-\theta \mathrm{v}\| \in\left[45^{\circ}, 135^{\circ}\right]$

$\Delta_{3},\|\theta s-\theta v\| \in\left[135^{\circ}, 180^{\circ}\right]$

(e) ${ }^{10^{-7}}$

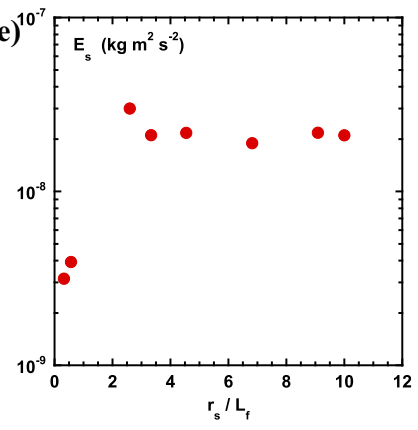

(c)

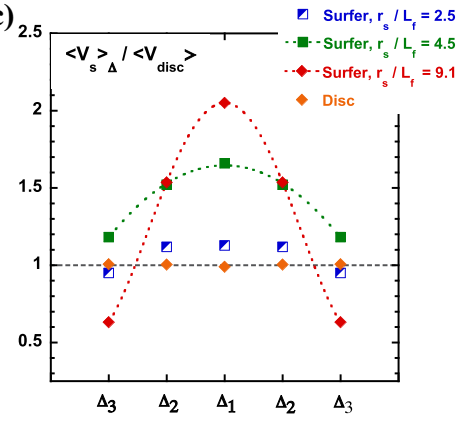

(f)

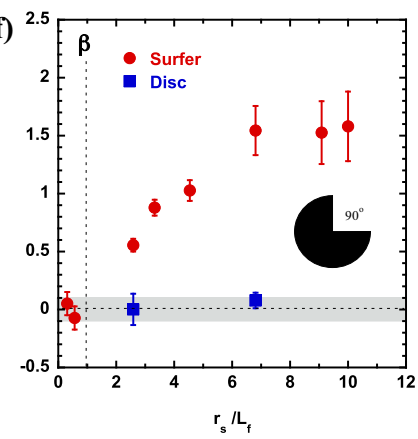

FIG. 5. (a) Probability density function of the angle difference $\delta \theta_{s v}=\theta_{s}-\theta_{v}$ for a disk and surfers with various values of the ratio $r_{s} / L_{f}$. (b) Schematics illustrating the definition of the three angular domains $\Delta_{i}$. The propulsive domain is $\Delta_{1}$, for which $\left\|\delta \theta_{s v}\right\| \in\left[0,+45^{\circ}\right]$; the turbulent domain is $\Delta_{2}$, for which $\left\|\delta \theta_{s v}\right\| \in$ $\left[45^{\circ}, 135^{\circ}\right]$; and the anticorrelated domain is $\Delta_{3}$, for which $\left\|\delta \theta_{s v}\right\| \in\left[135^{\circ}, 180^{\circ}\right]$. (c) Ratio $\left\langle\left|V_{s}\right|\right\rangle_{\Delta_{i}} /\left\langle\left|V_{\text {disc }}\right|\right\rangle$ as a function of the domain $\Delta_{i}$, where $\left\langle\left|V_{s}\right|\right\rangle_{\Delta_{i}}$ is the surfer velocity modulus conditionally averaged over an angular domain $\Delta_{i}$ and $\left\langle\left|V_{\text {disc }}\right|\right\rangle$ is the averaged velocity modulus of a disk of the same size as the surfer. (d) Mean-square velocity $\left\langle V_{s}^{2}\right\rangle$ of a surfer as a function of the size parameter $r_{s} / L_{f}$. (e) Kinetic energy $E_{s}=$ $M_{s}\left\langle V_{s}^{2}\right\rangle$ versus the parameter $r_{s} / L_{f}$ for a fixed turbulent flow energy $U^{2}$. (f) Propulsion parameter $\beta$ versus the scale ratio $r_{s} / L_{f}$. The parameter is defined as $\beta=\left(p_{1} V_{s 1}-p_{3} V_{s 3}\right) / p_{2} V_{s 2}$, where $p_{i}$ is the probability of $\left\|\delta \theta_{s v}\right\| \in \Delta_{i}$ and $V_{s i}=\left\langle\left|V_{s}\right|\right\rangle_{\Delta_{i}}$. The values of $\beta$ measured for disks, which theoretically should be zero, are also shown and used to indicate the noise level (gray area). The experimental parameters are $r_{s}=2.5-40 \mathrm{~mm}$, $U=2.3 \times 10^{-2} \mathrm{~ms}^{-1}$, and $L_{f}=3-9 \mathrm{~mm}$.

Surfers with size ratio $r_{s} / L_{f}<1$ do not show any sign of propulsion and $\beta \approx 0$. The parameter $\beta$ increases over the range $1<r_{s} / L_{f}<6$. Above $r_{s} / L_{f} \approx 6, \beta$ saturates at the level of about 1.5 ; in that regime the propulsion speed is 1.5 higher than the typical velocity component associated with the turbulent agitations.

As described in Ref. [18], the propulsion mechanism is related to the coupling of the riverlike structure of wave-driven turbulence with the surfer's boundary. In the vicinity of the surfer's corner, there is a fluid domain where these rivers are both protected from the external turbulent fluctuations and guided by the wall (see the schematics in Sec. 2 of Supplemental Material [29]). This guiding effect produces a reaction force that generates propulsion. The plateau in $\beta$ suggests that the size of this protected fluid domain producing the thrust cannot exceed $6 L_{f}$. This upper bound might be related to an important feature of laboratory 2D turbulence. In our experiments, the inverse cascade spreads energy up to scales close to $10 L_{f}$. This upper bound is readily observed since it determines the size of the largest domain in which spectral condensation, or the self-organization of 2D turbulence into large coherent structure, is observed experimentally [24,37]. The main ingredient for both spectral condensation and turbulence-driven propulsion is the confinement (global or partial) of $2 \mathrm{D}$ turbulence. 

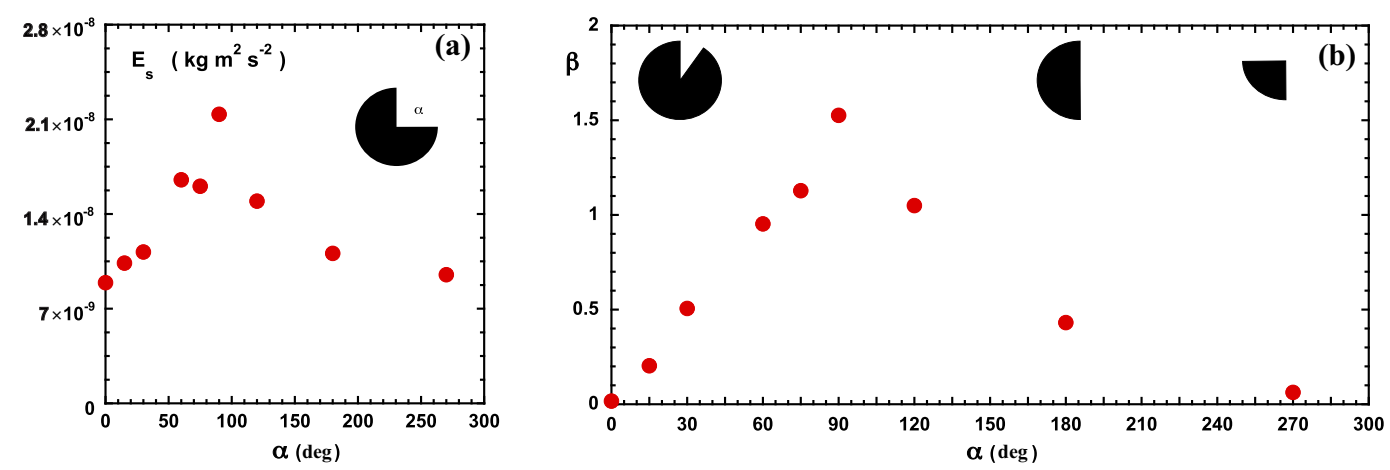

FIG. 6. Influence of the surfer's geometry on the propulsion. (a) Kinetic energy $E_{s}=M_{s}\left\langle V_{s}^{2}\right\rangle$ as a function of the angle $\alpha$ for a fixed turbulent flow energy $U^{2}$. (b) Propulsion parameter $\beta$ versus the angle $\alpha$. The experimental parameters are $r_{s}=40 \mathrm{~mm}, U=2.3 \times 10^{-2} \mathrm{~ms}^{-1}$, and $L_{f}=4.4 \mathrm{~mm}$.

The previous results concern the propulsion of a surfer with a fixed geometry. It is interesting to ask whether an optimal shape for the surfer exists for which both the extracted energy $E_{s}$ and the propulsion parameter $\beta$ will be maximum. To address this question, experiments were performed using surfers with different geometries. Given the complexity of the question, we restricted ourselves to the case of surfers with different angle $\alpha$ for the cut-out sector. In these experiments, the turbulent flow energy and forcing scale are fixed and we focus on the behavior of large surfers $r_{s} / L_{f} \approx 9$. Figure 6(a) shows the evolution of the surfer's kinetic energy $E_{s}$ over a broad range of angle $\alpha$. It reveals the existence of a maximum for $\alpha=90^{\circ}$. The energy extracted from turbulence by a surfer with a $90^{\circ}$ cut-out sector is two times larger than that of a disk or a wedge $\left(\alpha=270^{\circ}\right)$ with similar radius. Figure 6(b) shows how the parameter $\beta$ is impacted by the shape of the surfer. The propulsion parameter is a nonmonotonic function of $\alpha$ and it reaches a maximum value of $\beta \approx 1.5$ at $\alpha=90^{\circ}$. The results demonstrate that the geometry of the surfer is a key parameter to enhance the propulsive force. This effect is discussed further in Sec. III. To conclude this section, we emphasize that $\beta$ encapsulates two features of the surfer's motion: the enhanced angular alignment between the surfer's velocity and its orientation $\theta_{s}$ [Fig. 5(a)] and its ability to tap efficiently the turbulent flow energy [Fig. 5(c)].

\section{Propulsive and diffusive length scales}

A surfer propels itself with a speed $V_{p}$ along a direction prescribed by its geometry. It has been shown in Sec. II B that this direction of motion is subject to turbulent rotational diffusion, which leads to a coupling between translation and rotation. In this section, we use a simple minimal model to describe the influence of this coupling on the MSD of a surfer with a cut-out $90^{\circ}$ sector.

A key parameter to model the behavior of the surfer is the propulsion length scale $L_{\beta}=V_{p} T_{\theta}$, where $V_{p}$ is the propulsion speed and $T_{\theta}$ is the characteristic timescale of turbulent rotational diffusion. It represents the average distance traveled by a surfer before its direction is randomly changed. This length scale characterizes the coupling between propulsion and rotation. Based on this observation, we propose to model the MSD $\left\langle\delta r(t)^{2}\right\rangle$ of a surfer as

$$
\left\langle\delta r^{2}(\Delta t)\right\rangle=2 D_{t} \Delta t+\frac{V_{p}^{2} T_{\theta}^{2}}{2}\left[\frac{2 \Delta t}{T_{\theta}}+e^{-2 \Delta t / T_{\theta}}-1\right],
$$

where $D_{t}$ is the translational diffusion coefficient, i.e., $D_{t}$ characterizes the turbulent transport, and 〈〉 again denotes statistical averaging. We note that equations of the form of Eq. (1) are generally found in the modeling of an object endowed with a directed propulsive mechanism and subject to a process that randomizes the direction of its motion (turbulence in our case). For instance, a 


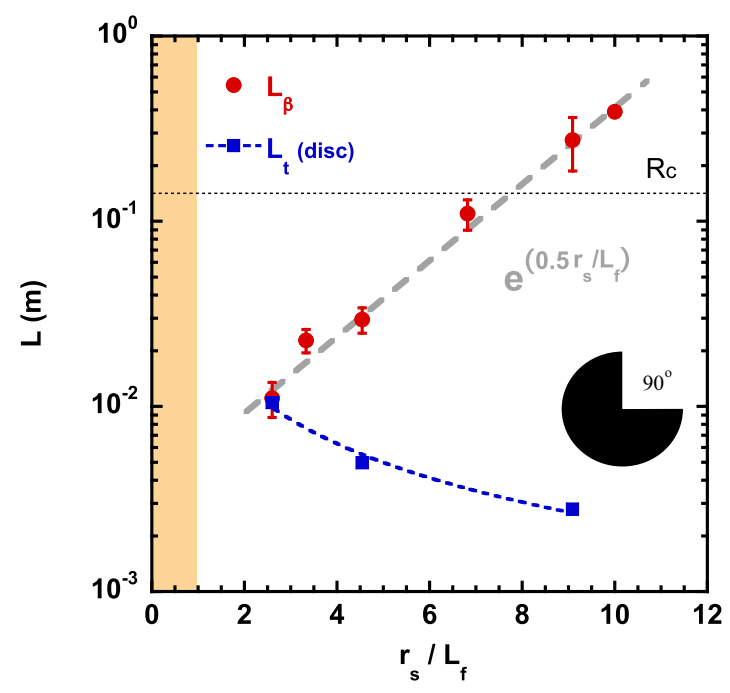

FIG. 7. Length scales $L_{\beta}$ and $L_{t}$ versus the scale ratio $r_{s} / L_{f}$ for a surfer with cut-out $90^{\circ}$ sectors. Here $L_{\beta}=V_{p} T_{\theta}$ is the propulsion length scale traveled by a surfer before its direction is randomized by the turbulence, while $L_{t}$ is the characteristic step size of the turbulent diffusive motion measured for a disk. The light gray dashed line indicates the exponential growth of $L_{\beta}$ versus $r_{s} / L_{f}$. The horizontal dashed line indicates the container radius $R_{c}$. The experimental parameters are $r_{s}=20-40 \mathrm{~mm}, U=2.3 \times 10^{-2} \mathrm{~ms}^{-1}$, and $L_{f}=$ 3-9 $\mathrm{mm}$.

similar model has been used to characterize the behavior of self-propelled colloidal particles whose propulsion mechanism relies on asymmetric chemical properties [38]. As shown in Figs. 3(c) and 3(d), Eq. (1) allows us to fit quite accurately our experimental measurements of $\left\langle\delta r^{2}(\Delta t)\right\rangle$.

An interesting aspect of the right-hand side of the equation is that it predicts a transition in the dynamics related to the characteristic timescale $T_{\theta}$. More precisely (if we neglect the diffusion coefficient $D_{t}$ for simplicity), two regimes can be described. (i) When $\Delta t<T_{\theta}$, the dynamics is dominated by $\left\langle\delta r^{2}(\Delta t)\right\rangle \approx L_{\beta}^{2} \Delta t^{2} / T_{\theta}^{2} \approx V_{p}^{2} \Delta t^{2}$, which corresponds to the ballistic propulsive regime. (ii) In the opposite limit, when $\Delta t>T_{\theta}$, we have $\left\langle\delta r^{2}(\Delta t)\right\rangle \approx L_{\beta}^{2} \Delta t / T_{\theta} \approx V_{p}^{2} T_{\theta} \Delta t$, which corresponds to the enhanced diffusion regime due to the coupling of rotational diffusion and propulsion.

In our experiments, Eq. (1) offers a basis for studying the interplay between three mechanisms: turbulent transport, propulsion, and rotational diffusion. The competition between these effects can be studied by comparing two characteristic length scales, namely, $L_{\beta}$ and a length scale associated with the turbulent transport. The latter can be defined as $L_{t}=2 D_{t} / u_{o}$, where $u_{o}$ is the rms velocity of a nonpropelling object, i.e., an object for which $V_{p}=0$. In our experiments, $L_{t}$ can be directly determined from the behavior of the floating disk, and we use $L_{t}=L_{\mathrm{disc}}=2 D_{\mathrm{disc}} / u_{\mathrm{disc}}$, where $u_{\text {disc }}$ is the rms velocity of the disk. The propulsion length scale $L_{\beta}$ can be estimated by using the measurements of $T_{\theta}$ (Sec. II B) and of the propulsion speed $V_{p}$ (Sec. II C). The length scale $L_{\beta}$ marks the transition from the propulsion-dominated regime to the enhanced diffusion regime; indeed, for $\Delta t=T_{\theta}$ the MSD is equal to $\left\langle\delta r^{2}\left(T_{\theta}\right)\right\rangle=L_{\beta}^{2}$. With this in mind, the comparison between $L_{t}$ and $L_{\beta}$ allows us to describe most of our previous observations. In particular, we can now quantitatively discuss the influence of the finite container size on our results.

Figure 7 shows the evolution of the characteristic length scales $L_{\beta}$ and $L_{t}$ versus the normalized size $r_{s} / L_{f}$. For the surfer, $L_{\beta}$ strongly increases with the increase in $r_{s} / L_{f}$ while for the disk $L_{t}$ decreases; $L_{\beta}$ shows an exponential dependence on $r_{s} / L_{f}$ which is inherited from the behavior of $T_{\theta}$ [Fig. 4(c)]. We note that $L_{t}$, the distance traveled by a disk before its motion becomes diffusive, 
is always much smaller than the container radius $R_{c}$. Therefore, for times long enough $\left[\Delta t>T_{\text {disc }}\right.$; see Fig. 2(d)], it is possible to observe the transition to the turbulent diffusion regime $\left\langle\delta r^{2}(\Delta t)\right\rangle \approx$ $2 D_{t} \Delta t$ irrespectively of the disk size.

For low values of the relative size $r_{s} / L_{f}<4$, a similar conclusion holds for a surfer: The longtime dynamics is diffusive because $L_{\beta}$ and $L_{t}$ have comparable magnitude and both are much smaller than the radius of the container $R_{c}$. In that regime, the effective diffusion coefficient of the surfer is actually composed of two terms $D=D_{t}+V_{p}^{2} T_{\theta}$.

However, when the size parameter is large $r_{s} / L_{f}>6, L_{\beta}$ becomes comparable to or larger than $R_{c}$ and much larger than $L_{t}$. For instance, $L_{\beta}$ is 100 times larger than $L_{t}$ and two times larger than $R_{c}$ for $r_{s} / L_{f}=9$.1. It implies that propulsion effects become dominant over the turbulent transport and that only the propulsive ballistic regime $\left\langle\delta r^{2}(\Delta t)\right\rangle \approx L_{\beta}^{2} \Delta t^{2} / T_{\theta}^{2}$ can be observed due to the finite size of the container.

An important insight given by this model is that, even for the largest surfers $\left(r_{s} / L_{f}>6\right)$, the propulsion mechanism described here actually will revert to a random walk for times longer than $T_{\theta}$ in an unbounded environment. In this ultimate regime, we note that the effective diffusion coefficient $D_{p}=V_{p}^{2} T_{\theta}$ is a strong function of the size parameter $r_{s} / L_{f}$ and it can be much higher than the turbulent diffusion coefficient $D_{t}$. For example, we find $V_{p}^{2} T_{\theta} \approx 500 D_{t}$ at $r_{s} / L_{f}=9.1$.

\section{DISCUSSION}

Recently, a physical mechanism which allows one to tap the kinetic energy accumulated in the inertial range of 2D turbulent flows was reported and the case of a turbulence-driven chiral rotor was studied in detail [18]. The Lagrangian nature of this mechanism of energy conversion raised the prospect to fuel not only rotation but also locomotion at a fluid surface. Here we have studied different aspects of such locomotion which is related to the broader topic of thrust generation in a flow. We demonstrate that asymmetric objects, referred to as surfers, can efficiently extract the energy of chaotic flows to fuel directed locomotion. The results show that the relative size of the object normalized by the flow forcing scale $r_{s} / L_{f}$ is a key parameter which controls the existence of this type of locomotion. The role played by the forcing scale $L_{f}$ in the propulsion mechanism is related to the coupling of the anisotropic object to the underlying riverlike structure of the turbulent flow. This coupling has been described in detail in [18] and it is the fuel of the propulsion force $F_{p}$ acting on the surfer (measurements of the force $F_{p}$ using an optical fiber cantilever [39-41] can be found in the Supplemental Material [29]). The mechanism relies on bundles of fluid trajectories of characteristic width $L_{f}$ that transfer their momentum to the surfer. The importance of this Lagrangian flow structure is reflected in the dependence of $V_{p}$ and $T_{\theta}$ on the scale ratio $r_{s} / L_{f}$; most importantly, passive propulsion is only observed when $r_{s} / L_{f}>1$.

This study reveal two key features of passive propulsion in wave-driven turbulence.

(i) The coupling of the translation and rotation of the surfer is a natural consequence of a directional propulsion mechanism related to the surfer's asymmetry. It implies that the characteristic timescale of the propulsion is the timescale $T_{\theta}$ associated with the rotational diffusion. It also implies that the motion of a surfer would ultimately revert to a random walk under the action of the turbulent rotational diffusion in an unbounded fluid domain. In our experiments, $T_{\theta}$ is an exponential function of the size parameter $r_{s} / L_{f}$. This feature gives us the ability to tune the magnitude of the effective diffusion coefficient $D_{p}=V_{p}^{2} T_{\theta}$ in a broad range with respect to the turbulent diffusion coefficient $D_{t}$.

(ii) The geometry of the surfer strongly impacts its locomotion. In these experiments, surfers are circular sectors and two geometric parameters were varied: the radius $r_{s}$ and the angle $\alpha$ of the cut-out sector. When the radius is varied and $\alpha$ is equal to $90^{\circ}$, it turns out that surfers extract the same amount of energy $E_{s}$ from the turbulent flow and the propulsion relies on the fraction of this energy that fuels the propulsion speed $V_{p}$. The larger the surfer, the better the conversion of the energy $E_{s}$ into directed motion. When the surfer is large $\left(r_{s} / L_{f}>1\right)$ and $\alpha$ is varied, an optimum shape for the passive locomotion has been identified; when $\alpha$ is equal to $90^{\circ}$, it maximizes both 
the kinetic energy $E_{S}$ harvested from the turbulent flow and the part of this energy that fuels the propulsion speed $V_{p}$.

The latter point suggests that efficient passive locomotion relies on a trade-off: On one hand, the angle $\alpha$ has to be large enough so that the surfer can interact with the turbulent eddies; on the other hand, a large $\alpha$ substantially lowers the moment of inertia and therefore will lead to increased angular fluctuations and less efficient propulsion. This trade-off is clearly captured by the behavior of $\beta$ versus $\alpha$ shown in Fig. 6(b).

In the context of exploiting the energy of turbulent flows, an interesting question concerns the estimate of the range of eddies that can efficiently fuel a propulsion mechanism. The question is of interest for both engineering applications (thrust generation by flow-structure coupling) and in biology (passive swimming) [2-4,13,14]. In these two domains, modeling approaches often describe the interaction of an object or a fish with eddies of comparable size among the hierarchy of turbulent eddies. These models assume some kind of localness of the transfer of energy (here local means that interactions mostly occur between the object and eddies of comparable size). Such a model would predict that larger surfers interact with large energy-containing eddies and can therefore harvest more energy from the flow. However, our results of Fig. 5(e) show that the extracted energy $E_{s}$ is constant over a broad range of surfer size.

Our understanding of the flow-surfer coupling is based on the characterization of the fine Lagrangian structure of 2D turbulence. More precisely, it was shown that a Kraichnan scaling $k^{-5 / 3}$ measured in the Eulerian frame can correspond to interacting riverlike structures in the Lagrangian frame. These rivers are elongated structures whose statistical length is much longer than their characteristic width $L_{f}$. This observation has consequences on the localness of the transfer of energy in wave-driven turbulence. Indeed, the forcing scale $L_{f}$ interacts with larger scales to give birth to the riverlike structures [18]. This shows that a level of nonlocality might exist in the transfer of energy. This also suggests that the coupling of the surfer to the flow fabric is also nonlocal and that in fact the surfers tap energy of the entire inertial interval in our experiments.

An interesting question concerns the extension of our results to $3 \mathrm{D}$ chaotic flows. The results presented in [18] show that passive propulsion is intrinsically connected to the Lagrangian structure of the flow (here bundles of fluid trajectories). It is possible that similar Lagrangian flow structures could exist in 3D disordered flows if there is a degree of anisotropy of the flow at small scales. If this is the case, then a mechanism of passive propulsion similar to that reported here should in principle be possible. Recent developments in the analysis of coherent structures in turbulent flows should allow one to detect the presence of similar Lagrangian features in 3D flows.

The presented experiments reveal that passive propulsion is possible in strongly turbulent flows. More studies are needed to explore the different aspects of turbulence-driven locomotion. One of these aspects is the enhanced turbulent transport of anisotropic object via the coupling of the object's geometric shape with the Lagrangian fabric of turbulent flows.

\section{ACKNOWLEDGMENTS}

This work was supported by the Australian Research Council's Discovery Projects funding scheme (Grants No. DP150103468 and No. DP160100863). N.F. acknowledges support from the Australian Research Council's DECRA award (Grant No. DE160100742). H.X. acknowledges support from the Australian Research Council's Future Fellowship (Grant No. FT140100067).

[1] E. Guyon, J.-P. Hulin, L. Petit, and C. D. Mitescu, Physical Hydrodynamics (Oxford University Press, Oxford, 2001).

[2] D. J. C. MacKay, Sustainable Energy -Without the hot air (UIT Cambridge, Cambridge, 2009).

[3] M. Shelley and J. Zhang, Flapping and bending bodies interacting with fluid flows, Annu. Rev. Fluid Mech. 43, 449 (2011). 
[4] S. Michelin and O. Doare, Energy harvesting efficiency of piezoelectric flags in axial flows, J. Fluid Mech. 714, 489 (2013).

[5] M. J. Lighthill, Hydromechanics of aquatic animal propulsion, Annu. Rev. Fluid Mech. 1, 413 (1969).

[6] M. Gazzola, M. Argentina, and L. Mahadevan, Scaling macroscopic aquatic locomotion, Nat. Phys. 10, 758 (2014).

[7] J. C. Liao and A. Cotel, in Swimming Physiology of Fish: Towards Using Exercise to Farm a Fit Fish in Sustainable Aquaculture, edited by A. P. Palstra and J. V. Planas (Springer, Cham, 2013), pp. 109-127.

[8] F. E. Fish and G. V. Lauder, Passive and active flow control by swimming fishes and mammals, Annu. Rev. Fluid Mech. 38, 193 (2006).

[9] J. C. Liao, D. N. Beal, G. V. Lauder, and M. S. Triantafyllou, Fish exploiting vortices decrease muscle activity, Science 302, 1566 (2003).

[10] J. Zhang, S. Childress, A. Libchaber, and M. Shelley, Flexible filaments in a flowing soap film as a model for one-dimensional flags in a two-dimensional wind, Nature (London) 408, 835 (2000).

[11] U. Lacis, N. Brosse, F. Ingremeau, A. Mazzino, F. Lundell, H. Kellay, and S. Bagheri, Passive appendages generate drift through symmetry breaking, Nat. Commun. 5, 5310 (2014).

[12] S. Ramananarivo, R. Godoy-Diana, and B. Thiria, Passive elastic mechanism to mimic fish-muscle action in anguilliform swimming, J. R. Soc. Interface. 10, 20130667 (2013).

[13] J. J. Allen and A. J. Smits, Energy harvesting eel, J. Fluid. Struct. 15, 629 (2001).

[14] D. N. Beal, F. S. Hover, M. S. Triantafyllou, J. C. Liao, and G. V. Lauder, Passive propulsion in vortex wakes, J. Fluid Mech. 549, 385 (2006).

[15] J. C. Liao, A review of fish swimming mechanics and behavior in altered flows, Philos. Trans. R. Soc. B 362, 1973 (2007).

[16] F. Toschi and E. Bodenschatz, Lagrangian properties of particles in turbulence, Annu. Rev. Fluid Mech. 41, 375 (2009).

[17] G. A. Voth and A. Soldati, Anisotropic particles in turbulence, Annu. Rev. Fluid Mech. 49, 249 (2017).

[18] N. Francois, H. Xia, H. Punzmann, and M. Shats, Rectification of chaotic fluid motion in two-dimensional turbulence, Phys. Rev. Fluids 3, 124602 (2018).

[19] H. Xia, N. Francois, H. Punzmann, and M. Shats, Tunable diffusion in wave-driven two-dimensional turbulence, J. Fluid Mech. 865, 811 (2019).

[20] H. Xia, N. Francois, B. Faber, H. Punzmann, and M. Shats, Local anisotropy of laboratory twodimensional turbulence affects pair dispersion, Phys. Fluids 31, 025111 (2019).

[21] J. W. M. Bush and D. L. Hu, Walking on water: Biolocomotion at the interface, Annu. Rev. Fluid Mech. 38, 339 (2006).

[22] M. Faraday, On the forms and states assumed by fluids in contact with vibrating elastic surfaces, Philos. Trans. R. Soc. London 121, 299 (1831).

[23] R. Kraichnan, Inertial ranges in two-dimensional turbulence, Phys. Fluids 10, 1417 (1967).

[24] N. Francois, H. Xia, H. Punzmann, and M. Shats, Inverse Energy Cascade and Emergence of Large Coherent Vortices in Turbulence Driven by Faraday Waves, Phys. Rev. Lett. 110, 194501 (2013).

[25] A. von Kameke, F. Huhn, G. Fernández-García, A. P. Muñuzuri, and V. Pérez-Muñuzuri, Double Cascade Turbulence and Richardson Dispersion in a Horizontal Fluid Flow Induced by Faraday Waves, Phys. Rev. Lett. 107, 074502 (2011).

[26] H. Xia and N. Francois, Two-dimensional turbulence in three-dimensional flows, Phys. Fluids 29, 111107 (2017).

[27] N. Francois, H. Xia, H. Punzmann, S. Ramsden, and M. Shats, Three-Dimensional Fluid Motion in Faraday Waves: Creation of Vorticity and Generation of Two-Dimensional Turbulence, Phys. Rev. X 4, 021021 (2014).

[28] H. Xia, N. Francois, H. Punzmann, and M. Shats, Lagrangian scale of particle dispersion in turbulence, Nat. Commun. 4, 3013 (2013).

[29] See Supplemental Material at http://link.aps.org/supplemental/10.1103/PhysRevFluids.4.104608 for details on experimental methods, flow characterization, production of the floating objects, and measurements of the propulsive force using an optical fiber cantilever and videos of the motion of a disk and a surfer placed in wave-driven turbulence. 
[30] H. Punzmann, N. Francois, H. Xia, G. Falkovich, and M. Shats, Generation and reversal of surface flows by propagating waves, Nat. Phys. 10, 658 (2014).

[31] N. Francois, H. Xia, H. Punzmann, P. W. Fontana, and M. Shats, Wave-based liquid-interface metamaterials, Nat. Commun. 8, 14325 (2017).

[32] H. Xu, A. Pumir, G. Falkovich, E. Bodenschatz, M. Shats, H. Xia, N. Francois, and G. Boffetta, Flight crash events in turbulence, Proc. Natl. Acad. Sci. USA 111, 7558 (2014).

[33] H. Xia, N. Francois, H. Punzmann, and M. Shats, Taylor Particle Dispersion during Transition to Fully Developed Two-Dimensional Turbulence, Phys. Rev. Lett. 112, 104501 (2014).

[34] N. Francois, H. Xia, H. Punzmann, and M. Shats, Braid entropy of two-dimensional turbulence, Sci. Rep. 5, 18564 (2015).

[35] N. Francois, H. Xia, H. Punzmann, and M. Shats, Wave-particle interaction in the Faraday waves, Eur. Phys. J. E 38, 10 (2015).

[36] M. Allshouse and J. L. Thiffeault, Detecting coherent structures using braids, Physica D 241, 95 (2012).

[37] H. Xia, M. Shats, and G. Falkovich, Spectrally condensed turbulence in thin layers, Phys. Fluids 21, 125101 (2009).

[38] J. R. Howse, R. A. L. Jones, A. J. Ryan, T. Gough, R. Vafabakhsh, and R. Golestanian, Self-Motile Colloidal Particles: From Directed Propulsion to Random Walk, Phys. Rev. Lett. 99, 048102 (2007).

[39] H. Kellay, X.-1. Wu, and W. I. Goldburg, Experiments with Turbulent Soap Films, Phys. Rev. Lett. 74, 3975 (1995).

[40] N. François, D. Lasne, Y. Amarouchene, B. Lounis, and H. Kellay, Drag Enhancement with Polymers, Phys. Rev. Lett. 100, 018302 (2008).

[41] N. Francois, Y. Amarouchene, B. Lounis, and H. Kellay, Polymer conformations and hysteretic stresses in nonstationary flows of polymer solutions, Europhys. Lett. 86, 34002 (2009). 